\title{
Status of the Baikal-GVD experiment - 2017
}

\begin{abstract}
A.D. Avrorin ${ }^{a}$, A.V. Avrorin ${ }^{a}$, V.M. Aynutdinov ${ }^{a}{ }^{1}$, R. Bannash $^{g}$, I.A. Belolaptikov ${ }^{b}$, V.B. Brudanin ${ }^{b}$, N.M. Budnev ${ }^{c}$, I.A. Danilchenko ${ }^{a}$, G.V. Domogatsky, A.A.Doroshenko ${ }^{a}$, R. Dvornickýb,h, A.N. Dyachok ${ }^{b}$ Zh.-A.M. Dzhilkibaev $^{a}$, L. Fajt ${ }^{b, h, i}$, S.V. Fialkovsky, A.R. Gafarov' ${ }^{c}$, K.V. Golubkov ${ }^{a}$, T.I. Gress ${ }^{c}$, Z. Honz ${ }^{b}$, K.G.Kebkal ${ }^{g}$, O.G. Kebkal ${ }^{g}$, M.M. Kolbin ${ }^{b}$, K.V. Konischev ${ }^{b}$, A.V. Korobchenko ${ }^{b}$, A.P.Koshechkin ${ }^{a}$, F.K. Koshel ${ }^{a}$, A.V. Kozhin ${ }^{d}$, V.F. Kulepov ${ }^{e}$, D.A. Kuleshov', M.B.Milenin ${ }^{e}$, R.A.Mirgazov ${ }^{c}$, E.R. Osipova ${ }^{d}$, A.I. Panfilov ${ }^{a}$, L.V. Pan'kov ${ }^{c}$, D.P.Petukhov ${ }^{a}$, E.N.Pliskovsky ${ }^{b}$, M.I., Rozanov ${ }^{f}$, E.V. Rjabov ${ }^{c}$, G.B.Safronov ${ }^{b}$, B.A.Shaybonov ${ }^{b}$, M.D.Shelepov ${ }^{a}$, F. Simkovic ${ }^{b, h, i}$, A.V. Skurikhin ${ }^{d}$, I.StekI ${ }^{i}$, O.V. Suvorova ${ }^{a}$, V.A., Tabolenko ${ }^{c}$, B.A.Tarashansky ${ }^{c}$, S.A.Yakovlev ${ }^{g}$, A.V.Zagorodnikov $^{c}$ and V.L. Zurbanov ${ }^{c}$
\end{abstract}

\author{
${ }^{a}$ Institute for Nuclear Research, Moscow, 117312 Russia \\ ${ }^{b}$ Joint Institute for Nuclear Research, Dubna, 141980 Russia \\ ${ }^{c}$ Irkutsk State University, Irkutsk, 664003 Russia \\ ${ }^{d}$ Institute of Nuclear Physics, Moscow State University, Moscow, 119991 Russia \\ ${ }^{e}$ Nizhni Novgorod State Technical University, Nizhni Novgorod, 603950 Russia \\ ${ }^{f}$ St. Petersburg State Marine Technical University, St. Petersburg, 190008 Russia \\ ${ }^{g}$ EvoLogics, Germany \\ ${ }^{h}$ Comenius University, Bratislava, Slovakia \\ ${ }^{i}$ Czech Technical University in Prague, Prague, Czech Republic
}

E-mail:aynutdin@yandex.ru

\begin{abstract}
Baikal-GVD is a kilometer scale neutrino telescope under construction in Lake Baikal, which will be formed by multimegaton subarrays - clusters of strings. First demonstration cluster "Dubna" has been deployed in 2015 and comprises 192 optical modules (OMs). In 2016 cluster "Dubna" was upgraded to baseline configuration which comprises $288 \mathrm{OMs}$ arranged at eight strings. The second full scale GVD-cluster was deployed and put in operation in 2017 in Lake Baikal. We review a present activity towards the GVD implementation and discuss some selected results obtained with array "Dubna".
\end{abstract}

35th International Cosmic Ray Conference - ICRC2017

10-20 July, 2017

Bexco, Busan, Korea

\footnotetext{
${ }^{1}$ Speaker

(C) Copyright owned by the author(s) under the terms of the Creative Commons 


\section{Introduction}

Deep underwater neutrino telescope Baikal-GVD [1] is designed to detect astrophysical neutrino fluxes at energies from a few $\mathrm{TeV}$ up to the highest energies of $10^{6} \mathrm{TeV}$ and particularly mapping the high-energy neutrino sky in the Southern Hemisphere including the region of the galactic center. The site chosen for the experiment is in the southern basin of Lake Baikal. Here, the combination of hydrological, hydro-physical, and landscape factors is optimal for deployment and operation of the neutrino telescope. The water depth is about $1360 \mathrm{~m}$ at distances beginning from about of three kilometers from the shore. The water transparency is characterized by an absorption length of about $20-25 \mathrm{~m}$ and a scattering length of $30-50 \mathrm{~m}$. The water luminescence is moderate at the detector site.

The development of a $\mathrm{km}^{3}$-scale neutrino telescope Baikal-GVD is carried out on the basis of long-term experience of the detector NT200 operation in Lake Baikal [2]. The first field tests of a new registration system of the Baikal-GVD were started in 2008 in condition of a combined operation with the NT200. A various versions of autonomous strings of Baikal-GVD were tested and fine-tuned in the period 2009 - 2012 [3]. The design of Baikal-GVD was substantially completed at 2013 [4] and a phased deployment of detector was launched. To date, the Baikal neutrino telescope includes the spatial structure of the 576 optical modules with effective volume for high energy cascades about $0.1 \mathrm{~km}^{3}$.

\section{Neutrino telescope Baikal-GVD}

The concept of Baikal-GVD is based on a number of evident requirements for the design and organization of the measuring system of the new detector: the utmost use of the advantages of mounting the telescope from the ice cover of Lake Baikal, the expandability of the system and ensure its effective operation already at the first stages of deployment, and the possibility of implementing different versions of arrangement and spatial density of photodetector location within the same measurement system.

Main detection units of the Baikal-GVD it is the optical modules (OM) [5] equipped with photomultiplier tubes Hamamatsu R7081-100 with photocathode diameter of 10" and with a quantum efficiency of $\sim 35 \%$. Optical modules are mounted on vertical load-carrying cables, forming strings which are fixed to the bottom with anchors. The choice of this approach to construction of measuring system is due to the method of deployment of the installation with surface of ice. The strings are grouped into clusters. Each cluster is a full functional detector which is capable of detecting a physical event both standalone mode, and as part of a full-scale installation. Underwater modules with the electronics that manages the cluster operation (Cluster $D A Q$ Center) are set close to the water surface (depth $\sim 25 \mathrm{~m}$ ). The Cluster DAQ Center is connected to the Shore DAQ Center by hybrid electro-optical cable.

A full-scale neutrino telescope Baikal-GVD will consist of array of about $10^{4}$ optical modules with instrumental volume of about $\sim 2 \mathrm{~km}^{3}$. The first phase of the installation (GVD-1) will be completed by 2020. GVD-1 will comprise of 8 clusters with about $2.3 \times 10^{3}$ OMs in total. Figure 2.1 shows the block diagram of the string, the view of cluster Baikal-GVD, and the layout of the GVD-1. The triangles on the plan show deep-water stations of the strings, circles indicate cable stations installed to date. 
Each cluster Baikal-GVD consists of 8 strings comprising 36 optical modules each. Distance between OMs along string is 15 meters. The bottom optical module of the string is placed at a depth of 1275 meters (about $100 \mathrm{~m}$ above the bottom of the lake), top OM is 750 meters below the lake surface. The distances between the strings in the cluster are $60 \mathrm{~m}$, the distances between the centers of the clusters are $300 \mathrm{~m}$.
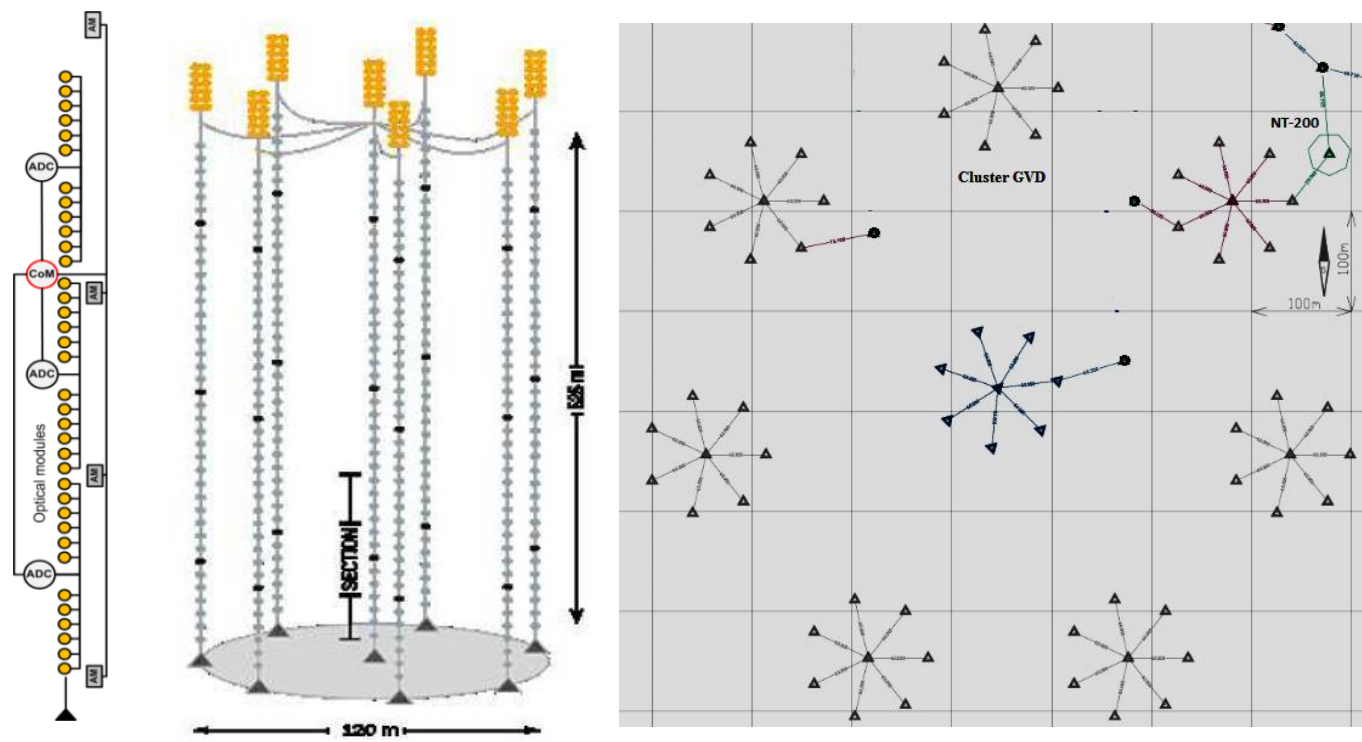

Fig. 2.1. Block diagram of the string and view of the cluster Baikal-GVD (left), and layout of eight clusters of the GVD-1 (right).

Neutrino telescope Baikal-GVD is intended for registration of the products of neutrino interaction, muons and cascade showers, in the energy range from $\sim 1 \mathrm{TeV}$ up to $10^{6} \mathrm{TeV}$. Figure 2.2 shows the effective volume for cascades and the effective area for muons of the installation for two configurations of the telescope: GVD-1 and full-scale neutrino telescope Baikal-GVD (GVD-4).
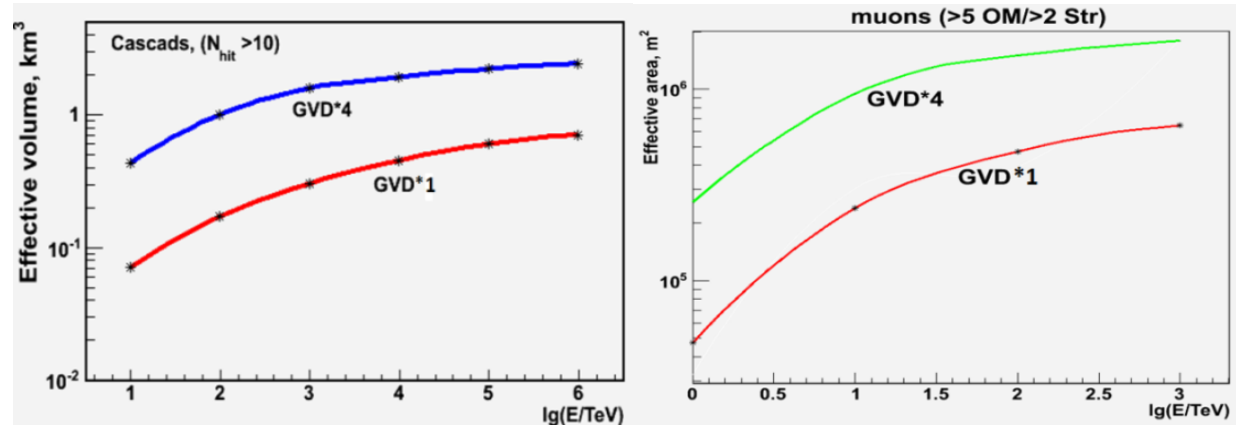

Fig. 2.2. The effective volume for the registration of the cascades and the effective area for muons depending on the energy for GVD-1 and full-scale neutrino telescope Baikal-GVD (GVD-4).

\section{Data acquisition system}

The basic structural unit of a data acquisition system of Baikal-GVD [6, 7] is a section of the optical modules. A section is a functionally complete unit that includes systems of registration of radiation, calibration systems, and control electronics for the formation of trigger, the signal processing, and data transfer. Three sections of optical modules reside on the same 
carrying cable form a string. Configuration of the section, which is currently the basis for the creation of the telescope, includes 12 optical modules with analog outputs, and a Central section Module (CM), which converts analog signals into a digital code. CM is located in the middle of the section, to minimize the lengths of cables connecting it to OMs. Each measuring channel of the section consists of PMT, preamplifier and 12-bit ADC with a sampling frequency of 200 $\mathrm{MHz}$ and an amplitude resolution of $1.6 \mathrm{mV}$. The conversion coefficients of the channels are leveled off an about $10^{8}$ by adjusting the high voltage of a PMT in the range from 1100 to 1800 $\mathrm{V}$. This provides the average single-photoelectron amplitude of the channels $\sim 25$ ADC counts.

The grouping OMs into separate sections allows to organize inter-module coincidences for suppressing of background glow of Baikal water. Coincidences of signals from any pairs of neighboring OMs with low threshold (0.5 - 1.5 p.e.) and high threshold (3 - 4 p.e.) are used as a local trigger of the section (signal request). Average frequencies of the section request signals are about $2-10 \mathrm{~Hz}$ in dependence on thresholds and water luminescence.

The request signals from three sections are combined in the Control Module of the string $(\mathrm{CoM})$ and transferred to the cluster DAQ center, where a global trigger is formed (signal acknowledge). Signal acknowledge, returning to each section of the cluster, stops the ADCs and initiates the formation of a master records of the sections and data transmitting to the Cluster DAQ center. Each master record comprises of the time of the trigger, the state of the counter of the acknowledge signals (used to merge records of the same event from different sections), and waveform for all 12 channels recorded in a time window of $5 \mu \mathrm{s}$ (1024 ADC time counts). Full length of master record is $12 \times 2048$ bytes. The total frequency of acknowledge signals is $50-$ $250 \mathrm{~Hz}$ (the sum of the frequencies of requests signals all sections of the cluster). Master records are transmitted to the Cluster center by the Ethernet network on the basis of Ethernet extenders with transfer rate up to $10 \mathrm{Mbit} / \mathrm{s}$. A relatively low bandwidth of network does not allow to transferring master records with full record size. So, master records are converted: a part of the records are selected in which ADC data exceeds a pedestal on the magnitude of $\sim 0.3$ single-photoelectron signal. Conversion process is implemented at the hardware level. The average size of the converted master record is approximately 300 bytes for basic mode of detector operation (muon detection). This ensures reliable transmission of full event flow.

In addition to the electronic units directly engaged in the registration process, data acquisition system includes a number of auxiliary subsystems: control of power supply, calibration of the detector, measuring of the coordinates of the optical modules. The system of power control allows disabling, if necessary, any of the underwater modules of the installation. Managed power switches, installed in the center of the cluster, in each string and in each section are used for these purposes. Calibration setup comprises LED light sources installed in each optical module (for amplitude and time calibration of the channels) and separate underwater modules with LEDs (for time calibration of the sections). Laser light source is used for the calibration of the cluster as a whole.

The coordinates of the optical modules are determined using an acoustic positioning system. Acoustic positioning system of the cluster comprises 32 acoustic modems (AM). Four AMs are mounted on each string on the distances from of the string bottom 1, 181, 346, and 538 meters. The coordinates of the AMs are measured with an accuracy of $\sim 2 \mathrm{~cm}$. Linear interpolation is used to determine the coordinates of the optical modules located between acoustic modems 


\section{The configuration of Baikal-GVD 2017}

The modular structure of the neutrino telescope Baikal-GVD provides the ability to conduct physical research in the early stages of the detector construction using different configurations of the installation. The stages of deployment a neutrino telescope Baikal-GVD and the corresponding configuration of the measuring system are presented in Table 4.1. The first cluster consisting of 8 strings with 24 OMs on each (two sections) was put into operation in 2015 [8]. The first cluster was upgraded to baseline configuration (three sections on the string) in 2016. The second cluster Baikal-GVD was commissioned in April 2017.

Table 4.1. Stages of deployment of the Baikal-GVD

\begin{tabular}{|l|c|c|c|}
\hline Configuration of the detector & 2015 & 2016 & 2017 \\
\hline The number of optical modules & 192 & 288 & 576 \\
\hline Geometric sizes & $\varnothing 40 \mathrm{~m} \times 345 \mathrm{~m}$ & $\varnothing 60 \mathrm{~m} \times 525 \mathrm{~m}$ & $2 \times \varnothing 60 \mathrm{~m} \times 525 \mathrm{~m}$ \\
\hline Effective volume $\left(\mathrm{E}_{\mathrm{sh}}>100 \mathrm{TeV}\right)$ & $0.03 \mathrm{~km}^{3}$ & $0.05 \mathrm{~km}^{3}$ & $0.1 \mathrm{~km}^{3}$ \\
\hline
\end{tabular}

Two full-scale clusters of Baikal-GVD were installed in Lake Baikal and are successfully operating now. Figure 4.1 shows the present layout of the installation and accumulative number of the master records for two clusters.
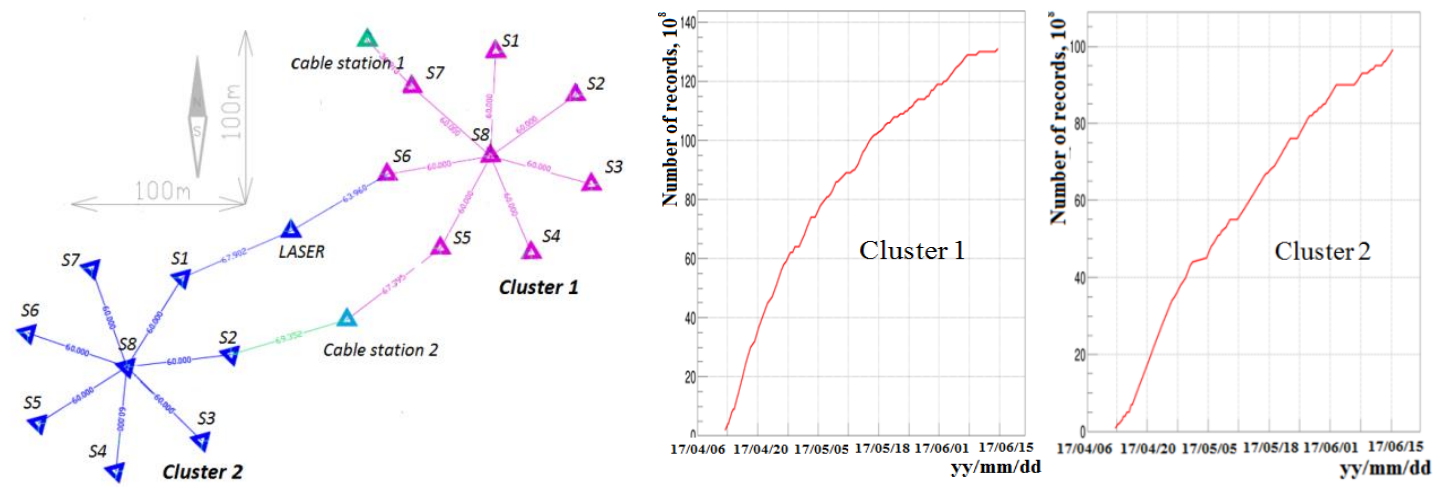

Fig. 4.1. Layout of the installation and accumulative number of the master records detected with two clusters (since April 13 to June 15 of 2017).

Each cluster consists of 8 Strings $(S 1 \ldots S 8)$ and the cluster $D A Q$ center, located on the central string ( $S 8$ ). Cluster $D A Q$ centers are connected to Shore center by hybrid opto-electrical cables providing their power supply (300 VDC) and data transmission. Additional cable stations are installed to raise the cables on the desired level from the lake bottom ( 25 meters below the lake surface) and to connect them to the Cluster DAQ centers (see Fig. 3.1). Laser calibration source is mounted on a separate station (Laser string) between two clusters. Two additional acoustic modems are installed on the laser string to measure its coordinates.

The deployment of the first cluster Baikal-GVD was carried out in stages, simultaneously with the debugging and upgrading of the equipment of data acquisition system. In particular, it includes different versions of the ADC boards. Currently, two ADC boards of the first version work unstable, which led to the need to exclude 24 channels from the configuration. All ADC boards of the second version, which used in the second cluster, are operating reliably now.

Table 4.2 shows the statistics of the clusters operation in the period from April 13 to 
June 15,2017 . About $10^{7}$ events containing more than $3 \times 10^{8}$ master records were detected for two months. The efficiency of the detector operation was about $85 \%$.

Table 4.2. Statistic of two clusters operation since April 13 up to June 15 of 2017.

\begin{tabular}{|c|c|c|c|}
\hline Number of RUNs & Duration of the work, days & Efficiency, \% & Master records \\
\hline Cluster-1: 130 & 64.3 & 84.4 & $1.87 \times 10^{8}$ \\
\hline Cluster-2: 117 & 64.0 & 87.6 & $1.45 \times 10^{8}$ \\
\hline
\end{tabular}

The first tests of a new laser calibration source were carried out during this period. The laser emits at a wavelength of $532 \mathrm{~nm}$, the pulse energy reaches a value of $0.37 \mathrm{~mJ}\left(\sim 10^{15}\right.$ photons), flash duration is about $1 \mathrm{~ns}$. A light diffuser is installed at the output of the laser beam.

The laser is installed at a level equals to the depth of the center of the lower sections. An example of the laser event detected with Cluster-1 is presented in Fig. 4.2. The dots show the time of registration of the laser pulse by the detector channels. Time calibration of the channels was provided by an independent method using LED calibration sources of the cluster. The numbering of the channels starts with the bottom OM of the first string: from 1 to 288 . The solid lines show the expected time obtained from calculation of light propagation from laser source to the strings. Laser flashes are reliably detected by all channels of the lower and middle sections of the strings. The lack of data on the second section is due to the fact that two sections were excluded from the configuration.

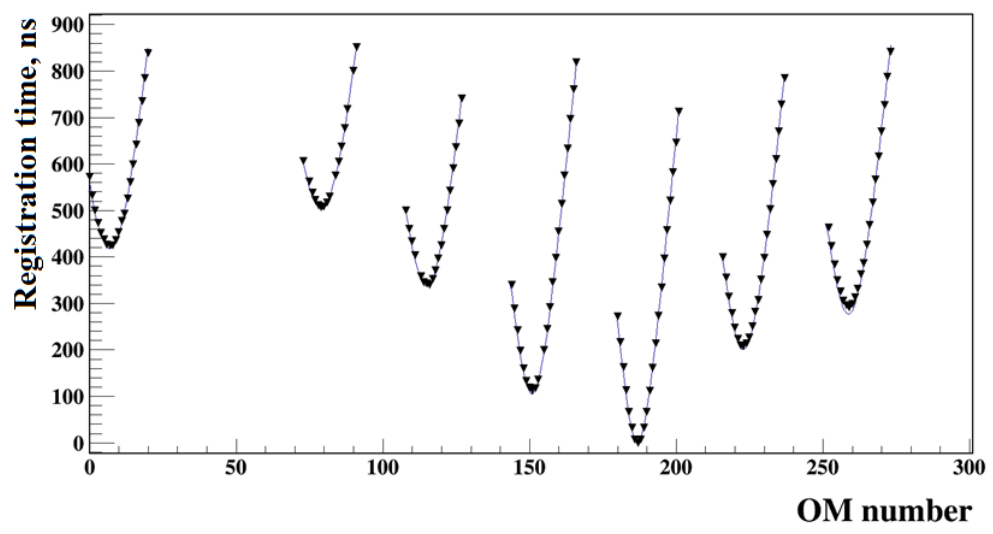

Fig. 4.2. An example of a laser event detected with the cluster of Baikal-GVD.

The expected time of the signals were calculated using the information about string and laser positions that provided by acoustic positioning system. It should be noted that while the bottom of the strings is rigidly fixed at the bottom of the lake by anchors, the top remains no fixed. This leads to significant deviations of the string from the vertical position due to the impact of currents of Lake Baikal. Fig. 4.3 shows the results of measurements of $x$ and $y$ coordinates of acoustic modems for different distances to anchors $z$. The measurements were performed continuously with a period of about 2 minutes during the whole time of the detector operation in 2017. The figure shows that with increasing height of the location of the modem $z$ maximum variation of their position in the $X-Y$ plane increases from $\sim 1 \mathrm{~m}$ to $\sim 10 \mathrm{~m}$. 

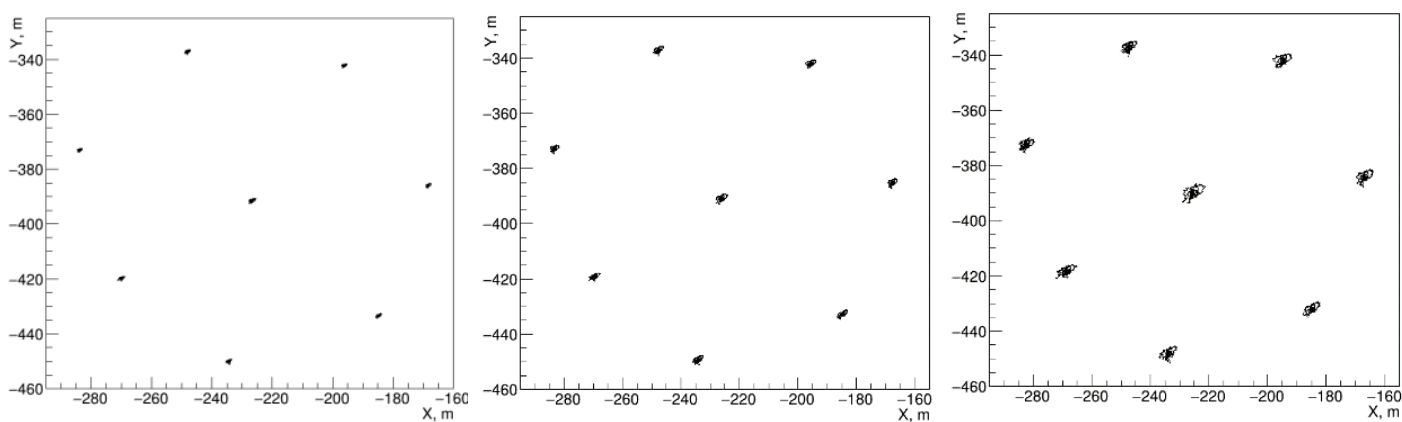

Fig. 4.3. The results of the measurement of the position of the acoustic modems installed on the eight strings of the cluster for different distances from AM to the string bottom: $z=181 \mathrm{~m}$ (left), $z=346$ (center), $z=538 \mathrm{~m}$ (right).

Good agreement between measured and expected time of registration of the laser flashes (see Fig. 4.2) indicates the correctness of the channel calibration and a sufficiently high positioning accuracy of the optical modules measured by the acoustic system.

\section{Cascade showers detection}

Registration of neutrino with the telescope Baikal-GVD is carried out by method of the detection of the products of their interactions: muons and cascade showers. A data sample obtained with the first cluster Baikal-GVD in the period from 24 Oct to 17 Dec 2015 was used for investigation and checking up of the methods of the selection from background and reconstruction of the cascade showers (41.6 days of the detector live time). About $4.4 \times 10^{8}$ triggered events were detected by the array during this time period. After applying several selection cuts and reconstruction of cascades vertex, energy and direction, 1192 events with reconstructed energy $\mathrm{E}>100 \mathrm{TeV}$ have been selected. Fig. 5.1 shows distribution of the selected 1192 events on multiplicity of the triggered channels. The experimental distribution is consistent with expectation from atmospheric muons for multiplicities $N_{h i t}<10$. The expected $N_{\text {hit }}$ distribution for events induced by atmospheric neutrinos and neutrinos of astrophysical nature (IceCube $E^{-2.46}$ neutrino spectrum) are also shown in Fig. 5.1.

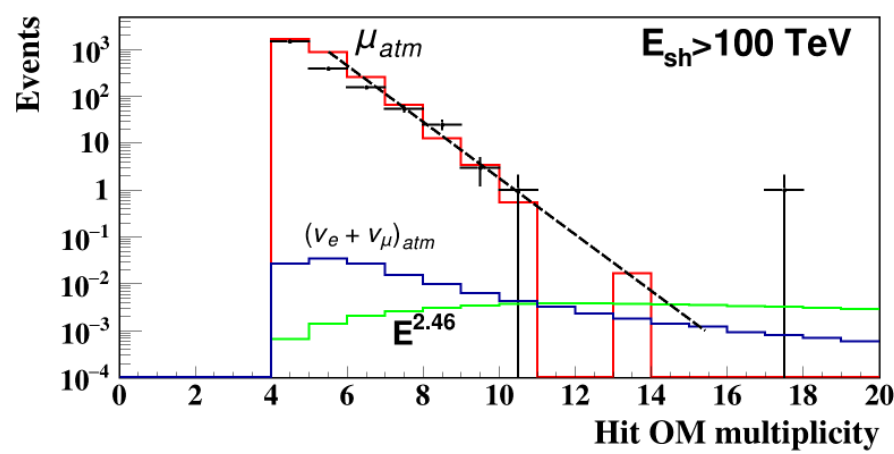

Fig. 5.1. The distribution of cascade-like events with energies above $100 \mathrm{TeV}$ on the multiplicity of hit channels; points - experiment, red line - background of atmospheric muons, blue line - background of atmospheric neutrinos, green line - expected signal from astrophysical neutrinos.

One event with $N_{h i t}=17$ was selected from the selected data sample. This event was reconstructed as high-energy cascade with energy of $107 \mathrm{TeV}$ and a zenith angle of $56.6^{\circ}$. The 
expected probability of event with $\mathrm{N}_{\text {hit }}>16$ is about $5 \%$ for astrophysical neutrino flux, and less than $1 \%$ for atmospheric neutrinos. More MC-simulation statistics is required for precise estimation of expected probability of such events for atmospheric muons. The presented methods of selection of the high energy cascades are currently used for the analysis of data recorded by the Baikal-GVD during 2016.

\section{Conclusion}

The ambition of the Baikal collaboration is to construct a $\mathrm{km}^{3}$-scale neutrino telescope: the Gigaton Volume Detector in Lake Baikal with implementation of about ten thousands photodetectors. The stage of prototyping of the GVD project has been performed since April 2011 and aims at comprehensive in situ test of all elements and systems of future detector. This phase was concluded in 2015 with the deployment and putting in operation of the first demonstration cluster "Dubna". In 2016 this array was upgraded to baseline configuration of GVD cluster with 288 OMs arranged on eight vertical strings. The second full-scale GVD cluster was installed and commissioned in April 2017. The operation of both clusters has been successfully tested with use of a new laser calibration source that was mounted in this year. The effective volume of a present GVD configuration for showers with energy above $100 \mathrm{TeV}$ is about $0.1 \mathrm{~km}^{3}$. The commissioning of the first stage of the Baikal neutrino telescope GVD-1 with effective volume $0.4 \mathrm{~km}^{3}$ is envisaged at 2020 .

The Baikal-GVD project is supported by the RFBR grants 16-29-13032, 17-02-01237.

\section{References}

[1] A.D. Avrorin et al., Baikal-GVD, EPJ Web Conf. 136, 04007 (2017).

[2] K. Antipin et al., Physics Results from the Baikal Neutrino Telescope, Nuclear Physics B (Proc. Suppl.) 168 (2007) 296-298.

[3] V. Aynutdinov, et al., The prototype string for the km3-scale Baikal neutrino telescope, NIM A602 (2009) 227-234.

[4] V. Aynutdinov et al., The prototyping/early construction phase of the BAIKAL-GVD project, NIM A742 (2014) 82-88.

[5] A.D. Avrorin et al., The optical module of Baikal-GVD, EPJ Web Conf. 116, 01003 (2016).

[6] A.V. Avrorin et. Al., Status of the Baikal-GVD project, NIM A 692 (2012) 46-52.

[7] V.M. Aynutdinov et al., The data acquisition system for Baikal-GVD, EPJ Web of Conf. 116, 05004(2016).

[8] A.D. Avrorin et al., Status of the early construction phase of Baikal-GVD, Nuclear and Particle Physics Proceedings 273-275 (2016) 314-320. 